\title{
The organic preservation effect of hemodilution
}

\author{
Do Xuan Hai', Mai Văn Viện²
}

'Department of Practical and Experimental Surgery, Military Medical University, Hanoi, Vietnam

${ }^{2}$ Department of Thoracic Surgery, 108 Medical Central Hospital, Hanoi, Vietnam

Background: Organ donation in countries with Buddhist cultures was developed primarily from living donor. Organs from the source for brain death with a low rate and the rate of organ transplantation eligible for transplantation account for 1.8 organs/ brain death donor. With the current multi-organ collection model, there are often hemodynamic abnormalities, which can cause arterial clotting, affecting the quality and function of the donor organ. This study aims to initially evaluate the multi-suberculosis method of blood dilution.

Methods: The study was conducted on experimental 30 pigs with the model of multi-organ blood dilution with the following steps: (1) animal preparation: pre-anesthesia with Atropine 1/4 mg (01 tube), Valium $10 \mathrm{mg}$ (01 tube), and Ketamine $100 \mathrm{mg}$; anesthesia with Pentothal at $60 \mathrm{mg} / \mathrm{kg}$ body weight. Use Arduan $4 \mathrm{mg}$ muscle relaxants and monitor over hemodynamic and central temperature. (2) Cause brain death in experimental animals using compression damage. (3) Place the cannula (20F) into the femoral artery (Scarpa triangle). Place two infusion tubes through the carotid vein and the thigh (7F). (4) Drop blood flowing slowly through the femoral cannula about $30 \%$ volume, simultaneously by intravenous (IV) infusion (Ringer lactate). Blood loss from $50 \%-60 \%$ began to appear hemodynamic disorders. Infuse IV Ringer lactate at $4^{\circ} \mathrm{C}$. Monitor pulse, blood pressure, and central temperature. (5) Blood flows in a stream through the femoral artery, quickly transfused through two veins (with phases Heparin and Kaleorid). Monitor temperature and hemodynamics until the animal stops heart. (6) Infuse fluids and organ preservative solutions continuously. Open the chest and abdomen, take the organs in blocks (heart-lung, liver-pancreas-intestine, kidney, etc.), and bring to the dissection table. Do pathological surgery to evaluate the quality of organs.

Results: Initial results showed that the central temperature decreased from $37.8^{\circ} \mathrm{C}$ to $32^{\circ} \mathrm{C}$, and heart rate and blood pressure were stable until $68 \%$ of the blood was withdrawn. Evaluation of donor organs: white color accounts for $29 / 30(96.67 \%)$, and white-pink accounts for $1 / 30(3.33 \%)$. The density of sure organs is $30 / 30(100 \%)$. The results of pathological anatomy: 360 samples showed that $10 / 360(2.7 \%)$ of the samples had microscopic lesions. Performing pancreatic, kidney, and lung transplant: having post-transplant function accounts for $100 \%$. The post-transplant survival rate followed up to 72 hours was $93.3 \%$ (14/15), $100 \%(15 / 15)$, and $93.3 \%(14 / 15)$.

Conclusions: Initial results show that this is an effective multi-organ washing method.

Corresponding author: Do Xuan Hai

E-mail: bsdoxuanhai@yahoo.com.vn

(C) The Korean Society for Transplantation

This is an Open Access article distributed under the terms of the Creative Commons Attribution Non-Commercial License (http://creativecommons.org/licenses/by-nc/4.0/) which permits unrestricted non-commercial use, distribution, and reproduction in any medium, provided the original work is properly cited. 